\title{
Desarrollo de un escáner 3D de bajo costo implementado por el método de triangulación espacial
}

\author{
David Del Castillo Vivanco ${ }^{\text {a }}$, David Loza ${ }^{\text {a }}$, Byron Cortez ${ }^{\text {a }}$, Víctor Erazo ${ }^{\text {b }}$ \\ ${ }^{a}$ Departamento de Ciencias de la Energía y Mecánica/Carrera de Ingeniería Mecatrónica, Universidad de las Fuerzas \\ Armadas “ESPE”, Sangolquí, Ecuador \\ b Facultad de Ingeniería en Ciencias Aplicadas/Carrera de Ingeniería Mecatrónica, Universidad Técnica del Norte, Ibarra, \\ Ecuador \\ didel.venture16@gmail.com, dcloza@espe.edu.ec, vaerazo@utn.edu.ec bhcortez@espe.edu.ec
}

\begin{abstract}
Resumen-El artículo es la elaboración de un digitalizador 3D lo suficientemente robusto para fines didácticos-educativos con la mínima inversión posible e implementado con software libre. Se aplica un método activo sin contacto de triangulación espacial por contorno láser con mesa rotatoria; haciendo uso de visión artificial para la segmentación del contorno láser formado sobre la superficie del objeto; por medio de métodos matemáticos y de triangulación espacial se lleva los puntos bidimensionales del contorno en las capturas a un espacio tridimensional. El diseño del sistema integra los elementos constitutivos, principio de funcionamiento, automatización e interacción con el usuario en un producto final. Con ayuda del software libre especializado Meshlab se realiza la reconstrucción superficial del objeto escaneado.
\end{abstract}

Palabras Clave-escáner 3D, ingeniería inversa, reconstrucción superficial.

Abstract-The article is the development of a 3D digitizer robust enough for didactic-educational purposes with the minimum possible investment and implemented with free software. An active non-contact method of spatial triangulation is applied by laser contour with rotating table; making use of artificial vision for the segmentation of the laser contour formed on the surface of the object; by means of mathematical methods and spatial triangulation the two-dimensional points of the contour in the captures are taken to a three-dimensional space. The design of the system integrates the constituent elements, principle of operation, automation and interaction with the user in a final product. With the help of specialized free software Meshlab, the surface reconstruction of the scanned object is carried out.

Keywords-3D scanner, reverse engineering, surface reconstruction.

\section{INTRODUCCIÓN}

Un escáner 3D [1] es un dispositivo que analiza un objeto para obtener datos de su forma para reconstruir modelos digitales. Existen dos tipos de escáner 3D. Por contacto [2]: El objeto se analiza mediante el apoyo de un elemento palpador sobre la superficie del objeto. Sin Contacto [3]: Son escáneres que no requieren contacto con el objeto, los cuales se subdividen en activos y pasivos. Los pasivos no emiten ninguna clase de radiación, en su lugar recepta la radiación reflejada por el ambiente, otros pueden utilizar radiación infrarroja, o de tipo estereoscópicos. Los activos son aquellos que emiten algún tipo de señal (electromagnéticas, haz de luz, ondas de radio, rayos $\mathrm{X}$ o ultrasonidos), la cual sirve para la construcción geométrica de un objeto, los hay de varios tipos, por facilidades de diseño y por el propósito económico se escogió un método activo sin contacto con un emisor láser lineal (generación del contorno) y una webcam (captura de la información) para el prototipo. Una mesa rotatoria hace posible la adquisición de datos de los $360^{\circ}$ alrededor del objeto. Para la segmentación del contorno láser formado sobre la superficie del objeto se hace uso de visión artificial. El modelo matemático desarrollado permite la generación y almacenamiento de la nube de puntos para su posterior estudio y tratamiento con el software libre de mallado Meshlab [4].

\section{SISTEMA DE ESCANEADO}

Compuesto por dos elementos esenciales, la webcam que funciona como sensor y el emisor láser lineal encargado de generar un haz de luz sobre el objeto que se desea escanear.

En el mercado local es difícil la adquisición de un dispositivo láser lineal, por lo cual se optó por la fabricación de uno. La construcción del láser lineal constará de un diodo y un cilindro de vidrio (lente) de $\varnothing 3 \mathrm{~mm}$ para formar el haz de luz en forma de línea como se observa en la Fig. 1.

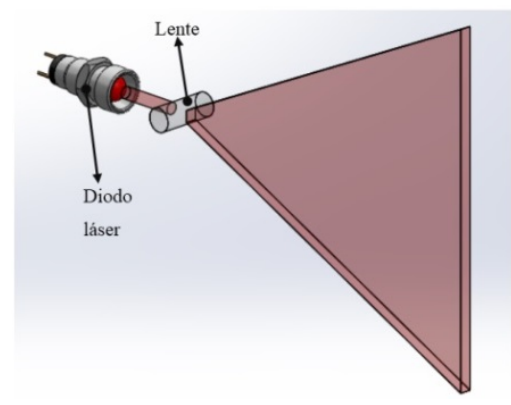

Fig. 1. Refracción del puntero láser.

La Tabla I muestra la etapa de configuración de parámetros de inicio, se habilitará al usuario por medio de una HMI la posibilidad de poder modificar las siguientes variables de control:

TABLA I

PARÁMETROS DE INICIO

\begin{tabular}{ll}
\hline \hline Variable & Descripción \\
\hline Ángulo de paso & Desplazamiento del paso del motor $3,6^{\circ}$ y $7,2^{\circ}$ \\
Salto de Pixel & 8 y 16 \\
$\begin{array}{l}\text { Sensibilidad de la } \\
\text { escala de rojo }\end{array}$ & $\begin{array}{l}100 \text { a } 255 \text { (valor } 140 \text { por defecto). Intensidad de } \\
\text { rojo detectable, a valores pequeños mayor } \\
\text { sensibilidad. }\end{array}$ \\
\hline \hline
\end{tabular}


De las 3 variables y sus combinaciones dependerá la calidad de escaneado y el tiempo de procesamiento. El subproceso Adquisición de Datos inicia con la captura de la imagen del objeto que se desea escanear.

Las imágenes digitalizadas forman arreglos matriciales de mxn pixeles [5], que al tratarse de pixeles a color se trabajará en una escala RGB de 1 byte por color. Se ejecuta tratamiento de la capa roja de cada captura [6], donde el algoritmo deberá separar las tres capas de color RGB y almacenar únicamente la roja.

El siguiente paso es segmentar la proyección del objeto producida por el láser, obteniendo las coordenadas $(\mathrm{x}, \mathrm{y})$ de la posición de los puntos que conforman el contorno dentro de la imagen. La reestructuración de los puntos obtenidos, en un espacio tridimensional es posible mediante la aplicación de (1).

$$
\left\{\begin{array}{l}
X_{\text {real }}=K p \cdot K t \cdot d u_{x} \cdot \cos \theta \\
Y_{\text {real }}=K p \cdot\left(d u_{y}+t r_{y}\right) \\
Z_{\text {real }}=-K p \cdot k t \cdot d u_{x} \cdot \sin \theta
\end{array}\right.
$$

donde $K t$ es el coeficiente de triangulación, $K p$ es el coeficiente de proporcionalidad, $d u_{x}$ es la distancia útil en "X" (distancia en pixeles de la línea centro de la imagen al contorno del objeto), $d u_{y}$ es la distancia útil en "Y" (distancia en pixeles de la parte inferior de la imagen al contorno del objeto), $t r_{y}$ es la traslación del punto de contorno en el eje "y" y $\theta$ es el ángulo de rotación.

Cada contorno escaneado del objeto debe ser rotado espacialmente en $\theta$ grados, por lo cual será prescindible la utilización de una matriz de rotación (2) sobre el eje "Y", donde $X^{\prime}, Y^{\prime}$ y $Z^{\prime}$ 'son las coordenadas de los puntos rotados en el espacio [7].

$$
\begin{gathered}
R(r, \phi)=\left[\begin{array}{ccc}
\cos \phi & 0 & \sin \phi \\
0 & 1 & 0 \\
-\sin \phi & 0 & \cos \phi
\end{array}\right] \\
\left(\begin{array}{l}
X^{\prime} \\
Y^{\prime} \\
Z^{\prime}
\end{array}\right)=R\left(\begin{array}{l}
X \\
Y \\
Z
\end{array}\right)=\left(\begin{array}{c}
X \cos \phi+Z \sin \phi \\
Y \\
-X \sin \phi+Z \cos \phi
\end{array}\right)
\end{gathered}
$$

Multiplicando la matriz $R$ por un punto (3), se obtiene el punto rotado $\phi$ grados sobre el eje "Y". El valor de $Z$ en la aplicación es siempre cero, debido a que el cálculo de cualquier punto rotado se lo hará desde un mismo eje de referencia. Quedando lo siguiente:

$$
\left(\begin{array}{c}
X^{\prime} \\
Y^{\prime} \\
Z^{\prime}
\end{array}\right)=\left(\begin{array}{c}
X \cos \phi \\
Y \\
-X \sin \phi
\end{array}\right)
$$

La traslación del punto de contorno en el eje "Y" es la agregación de una distancia en pixels $\left(t r_{y}\right)$ a la triangulación espacial de los puntos. Se requiere saber la cantidad de pixeles que debemos recorrer a los puntos escaneados (Ec. 5). La cantidad $t r_{y}$ dependerá de la distancia de cada punto a la línea centro de la imagen $d u_{x}$ y del ángulo $\theta$ formado por el desplazamiento que se requiere dar como se observa en la Fig. 2.

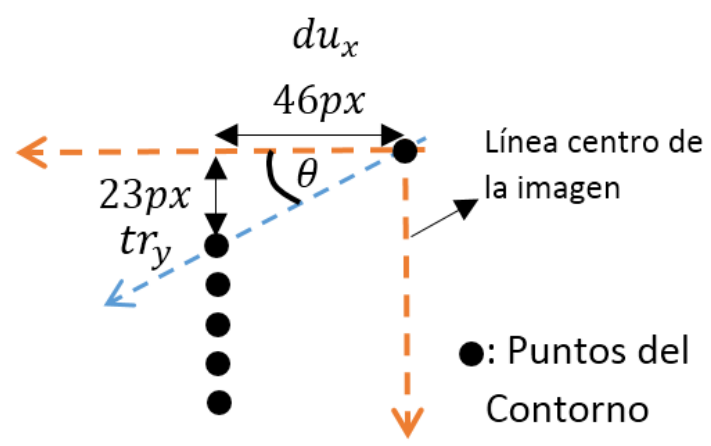

Fig. 2. Diagrama de traslación de pixeles.

Se obtiene:

$$
\begin{aligned}
& \tan \theta=\frac{t r_{y}}{d u_{x}} \\
& t r_{y}=d u_{x} \cdot 23 / 46 \\
& t r_{y}=0.5 \cdot d u_{x}
\end{aligned}
$$

El coeficiente de triangulación Kt (6), se calcula a partir del posicionamiento del láser, webcam y el objeto que se desea escanear, formando entre ellos un triángulo como se observa en la Fig. 3.

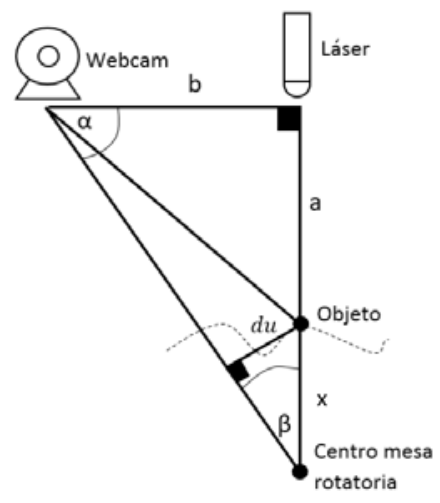

Fig. 3. Modelo de triangulación del prototipo

Donde $\mathrm{a}+\mathrm{x}=250 \mathrm{~mm}$ y b $=140 \mathrm{~mm}$ como constantes dimensionales del prototipo. Se desea conocer la relación entre $d u$ (distancia útil en pixeles) y $x$ (distancia real en pixeles), por ello se calculara el ángulo $\beta$ y así también hallar el coeficiente de triangulación.

Entonces:

$$
x=\frac{d u}{\sin \beta}, \quad K t=\frac{1}{\sin \beta}=2.05
$$

El coeficiente de proporcionalidad $K p$ describe la relación entre unidad de pixel y unidad milimétrica. Teniendo en cuenta la variación de pixeles en el eje "Y" de la imagen $\Delta p x_{y}$ y la altura del objeto escaneado, es sencillo deducir la relación que guardan estos valores. A continuación en (7) se demuestra la obtención del valor $K p$.

$$
K p=\frac{d_{\text {real }}}{\Delta p x_{y}}=\frac{160}{528}=0.3
$$

Escaneados los puntos del contorno de los $360^{\circ}$ del objeto, se almacenarán en una matriz de dimensión. Al mismo 
tiempo se genera un archivo que contenga la nube de puntos con la extensión “.asc” para su posterior procesamiento en el software especializado en el mallado de puntos.

\section{PRUEBAS Y RESULTADOS}

En la etapa de pruebas del prototipo, se dedujo que el coeficiente $K p$ es variable. Para determinar el valor se utilizó el siguiente algoritmo (8):

$$
K p^{\prime \prime}=K p^{\prime}\left(\frac{x}{187}\right)
$$

donde $x$ es el valor de la coordenada en " $x$ " del punto de contorno.

La primera prueba se realizó en un objeto cilíndrico, las dimensiones del cilindro son: $\phi 65 \mathrm{~mm}$ y $48.5 \mathrm{~mm}$ de altura. Los resultados obtenidos se describen en la Tabla IV.

TABLA II

RESULTADOS PRIMERA EVALUACIÓN

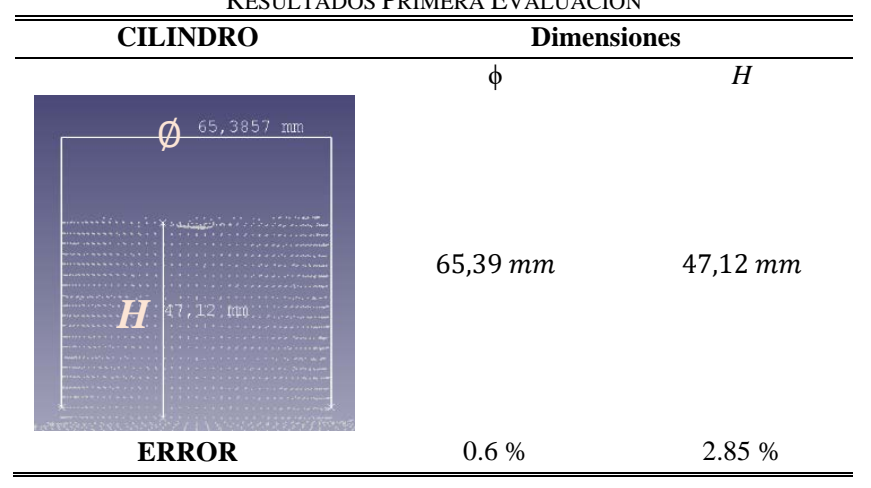

La segunda evaluación del funcionamiento del escáner se la realiza con un envase con dimensiones mostradas en la Fig. 4. Los resultados se describe en la Tabla V.

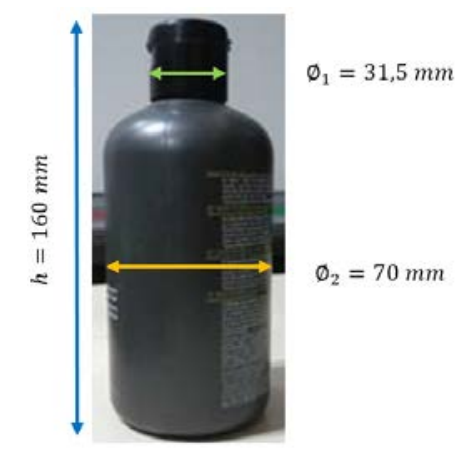

Fig. 4. Objeto de prueba (envase).

Los errores dimensionales obtenidos en la primera y segunda evaluación son inferiores al 5\%, alcanzando así un nivel aceptable de funcionamiento relacionado con la generación de la nube de puntos y su exactitud.

La tercera prueba evalúa la capacidad del escáner para digitalizar superficies cóncavo-convexas. En primer lugar se realiza una prueba métrica de las dimensiones del objeto escaneado, Fig. 5(a), comparando con el objeto real; el análisis se muestra a continuación en la Tabla VI.
TABLA III

RESULTADOS SEGUNDA EVALUACIÓN

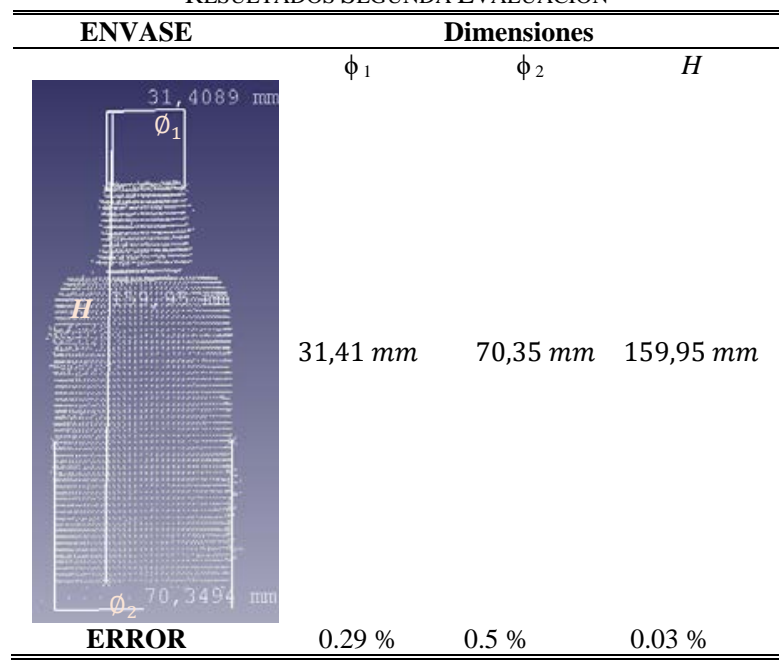

TABLA IV

Prueba Dimensional OBjeto CónCaVo-Convexo

\begin{tabular}{cccc}
\hline \hline Dimensión & Real & Escaneada & ERROR \\
\hline $\boldsymbol{h}$ & $100 \mathrm{~mm}$ & $99,57 \mathrm{~mm}$ & $E \%=0,43 \%$ \\
$\boldsymbol{a}$ & $100 \mathrm{~mm}$ & $99,89 \mathrm{~mm}$ & $E \%=0,11 \%$ \\
$\boldsymbol{b}$ & $100 \mathrm{~mm}$ & $99,94 \mathrm{~mm}$ & $E \%=0,06 \%$ \\
$\emptyset_{\mathbf{1}}$ & $60 \mathrm{~mm}$ & $59,34 \mathrm{~mm}$ & $E \%=1,1 \%$ \\
$\emptyset_{\mathbf{2}}$ & $60 \mathrm{~mm}$ & $59,28 \mathrm{~mm}$ & $E \%=1,2 \%$ \\
\hline \hline
\end{tabular}

Validadas las dimensiones se prosigue con la reconstrucción de la nube de puntos, Fig. 5(b). El método seleccionado para el mallado es Ball Pivoting por considerarse al objeto de cierta complejidad.

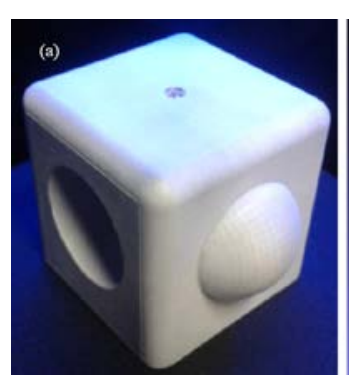

(a)

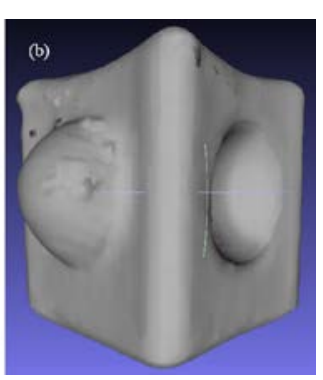

(b)
Fig. 5. Prueba de reconstrucción superficial objeto cóncavo-convexo (a) Pieza impresa y (b) Pieza mallada.

La última prueba no intenta medir errores dimensionales, sino más bien analizar la respuesta de digitalización del escáner 3D de bajo costo en tres tipos de figuras geométricas: Triángulos, círculos y cuadrados en diferentes tamaños.

El objeto de prueba (Fig. 7) es un prisma cuadrado de $50 \times 50 \times 150 \mathrm{~mm}$ que tiene en tres de sus caras verticales las figuras geométricas destinadas para el estudio. Se usa la denominación (Cir.) para los cilindros circulares de dimensión $\phi$, (Tri.) para las pirámides triangulares (el triángulo base es circunscrito en un círculo de diámetro $\phi$ ) y (Sqr.) para los cilindros cuadrados de lado $l$. 

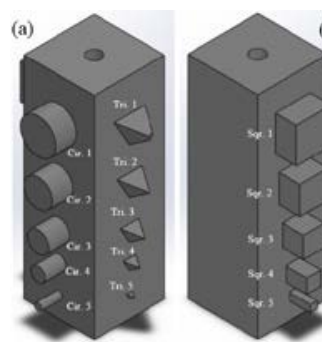

(a)

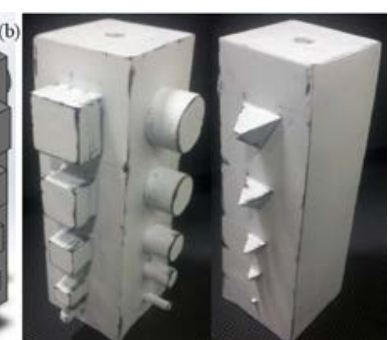

(b)

Fig. 6. Prueba de figuras geométricas (a) Diseño CAD y (b) pieza impresa.

En la Tabla VII se observa el resultado de la reconstrucción superficial del objeto de prueba de figuras geométricas que se realizó mediante el método de Ball Pivoting. Se analiza cada una de las figuras en los distintos tamaños para determinar si son legibles a simple vista o no.

TABLA V

Resultados De La Prueba De Figuras Geometricas

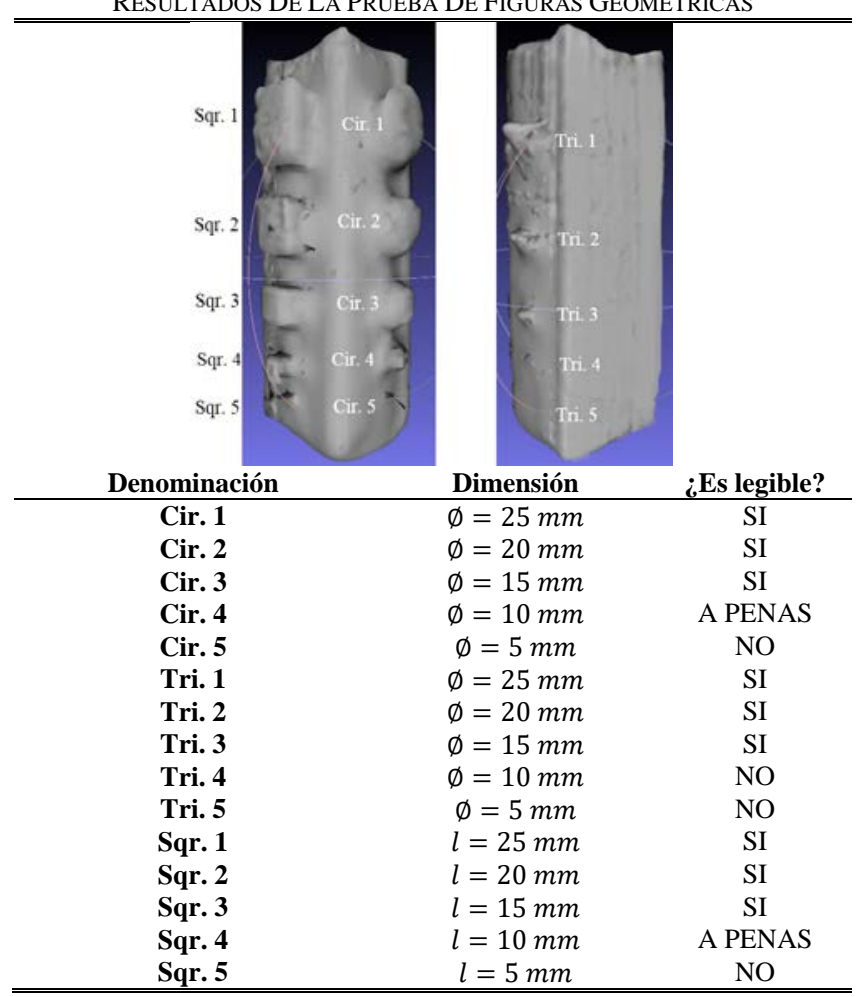

Se puede apreciar que las figuras geométricas más pequeñas son poco o nada legibles, se debe a parámetros de escaneo como el ángulo de paso del motor que no permite obtener los suficientes puntos de contorno para definir con mayor exactitud las figuras; la longitud de onda del láser lineal que debido a su anchura impide una segmentación fina del contorno generado y al salto de pixel que provoca perdida de información para salvaguardar el rendimiento en la generación de la nube de puntos.

\section{CONCLUSIONES}

Se diseñó y construyó un prototipo de escáner 3D de bajo costo con una inversión aproximada de 186 dólares. El uso de software libre contribuyo sustancialmente en lo que ha costos respecta. Los errores arrojados en la etapa de pruebas son menores al $5 \%$, esto se debe gracias a que el modelamiento

matemático para la triangulación espacial (formada por la webcam, emisor láser lineal y el contorno del objeto escaneado) fue correctamente realizada. Las especificaciones técnicas establecidas en la etapa de diseño ayudaron significativamente a satisfacer los requerimientos del prototipo. Es capaz de escanear objetos de $16 \times 16 \times 18 \mathrm{~cm}$ de hasta $6 \mathrm{~kg}$ de peso. Presta gran precisión de escaneo y permite el giro de los $360^{\circ}$ del objeto. El sistema implementado permite al usuario un fácil uso del prototipo mediante una interfaz amigable y ergonómica.

\section{AGRADECIMIENTOS}

A la Universidad de las Fuerzas Armadas "ESPE" por brindar la posibilidad de realizar el presente estudio y al Laboratorio de Procesos de Manufactura por facilitar los medios pertinentes para construcción del prototipo de escáner 3D de bajo costo.

\section{REFERENCIAS}

[1] I. B. Montero, Equipos Microinformáticos, Madrid, ESPAÑA: Ediciones Paraninfo SA, 2da Edición, 2016.

[2] MicroScribe, "MicroScribe 3D G2X Portable CMM," 2016. [En línea]. Available: http://www.3d-microscribe.com/G2\%20Page.htm.

[3] C. Teutsch, "Model-based Analysis and Evaluation of point sets from 3D laser Scanners,” Shaker Verlag, Magdeburger Schriften zur, 2007.

[4] P. Cignoni, M. Callieri, M. Corsini, M. Dellepiane, F. Ganovelli y G. Ranzuglia, "MeshLab: an Open-Source Mesh Processing Tool,” 2008. [En línea]. Available: http://www.meshlab.net/.

[5] G. d. I. EDMANS, “Técnicas y Algoritmos básicos de visión artificial,” Universidad de la Rioja, 2006.

[6] A. B. M. D. Á. S. C. José Francisco Vélez, "Visión por Computador," 2003.

[7] J. J. Craig, Robótica-Matriz de rotación, 3era ed., PEARSON, pp. 4245.

[8] S. Fleishman, D. Cohen-Or y C. T. Silva, "Robust Moving Leastsquares Fitting with Sharp Features,” [En línea]. Available: http://www.sci.utah.edu/ shachar/Publications/rmls.pdf. [Último acceso: 2402 2017].

[9] W. E. Lorensen y H. E. Cline, "Marching Cubes: A high resolution 3D surface construction algorithm,” General Electric Company, New York, 1987.

[10] F. Bernrdini, J. Mittleman, H. Rushmeir, C. Silva y G. Taubin, "The Ball-Pivoting Algorithm for Surface Reconstruction,” IBM Research.

[11] M. Kazhdan, M. Bolitho y H. Hoppe, "Poisson Surface Reconstruction,” Microsoft Research, Redmond WA, USA, 2006.

[12] A. 3D, “Escáneres de Artec 3D \& Aplicaciones,” 2017. [En línea]. Available: https://www.artec3d.com/es/applications\#aplication-rowanchor-1.

[13] P. +. Fuchs, "PRT: El Método de Medición de Gran Precisión,” 2017. [En línea]. Available: www.pepperl-fuchs.es/spain/es/10090.htm.

[14] Optimet, "Holografía Conoscópica," [En línea]. Available: http://www.dsiplus.es/web/optimet.asp.

[15] P. H. Song Zhan, "High-resolution, real-time three-dimensional shape measurement,” 2006. 